\title{
Assessment of Methods to Consolidate lodine-Loaded Silver-Functionalized Silica Aerogel
}

Fuel Cycle Research \& Development

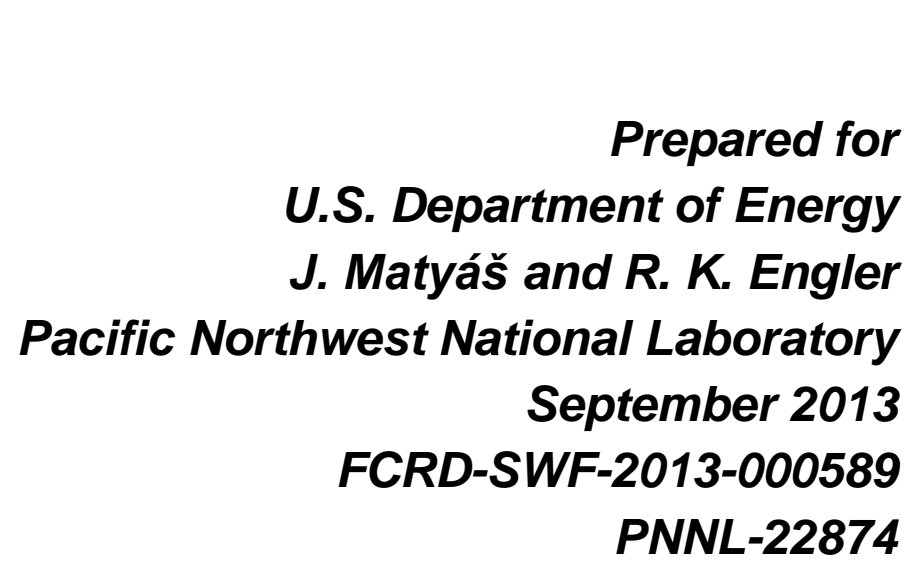




\section{DISCLAIMER}

This information was prepared as an account of work sponsored by an agency of the U.S. Government. Neither the U.S. Government nor any agency thereof, nor any of their employees, makes any warranty, expressed or implied, or assumes any legal liability or responsibility for the accuracy, completeness, or usefulness, of any information, apparatus, product, or process disclosed, or represents that its use would not infringe privately owned rights. References herein to any specific commercial product, process, or service by trade name, trade mark, manufacturer, or otherwise, does not necessarily constitute or imply its endorsement, recommendation, or favoring by the U.S. Government or any agency thereof. The views and opinions of authors expressed herein do not necessarily state or reflect those of the U.S. Government or any agency thereof. 


\section{SUMMARY}

The U.S. Department of Energy is currently investigating alternative sorbents for the removal and immobilization of radioiodine from the gas streams in a nuclear fuel reprocessing plant. One of these new sorbents, $\mathrm{Ag}^{0}$-functionalized silica aerogels, shows promise as a potential replacement for $\mathrm{Ag}$-bearing mordenites because of its high selectivity and sorption capacity for iodine. Moreover, a feasible consolidation of iodine-loaded $\mathrm{Ag}^{0}$-functionalized silica aerogels to a durable $\mathrm{SiO}_{2}$-based waste form makes this aerogel an attractive choice for sequestering radioiodine.

This report provides a preliminary assessment of the methods that can be used to consolidate iodineloaded $\mathrm{Ag}^{0}$-functionalized silica aerogels into a final waste form. In particular, it focuses on experimental investigation of densification of as-prepared $\mathrm{Ag}^{0}$-functionalized silica aerogel powders, with or without an organic moiety and with or without a sintering additive (colloidal silica), with three commercially available techniques: 1) hot uniaxial pressing (HUP), 2) hot isostatic pressing (HIP), and 3) spark plasma sintering (SPS). Since there are no studies reported in literature on the sintering of aerogels with HIP and SPS, the preliminary experiments were performed without iodine-loaded aerogels and were focused on investigating feasibility of these methods to produce a fully dense product. Iodine was excluded from these initial studies to avoid potential damage to vendor equipment due to the potential release of iodine during consolidation. The densified products were evaluated with a helium gas pycnometer for apparent density, with the Archimedes method for apparent density and open porosity, and with high-resolution scanning electron microscopy and energy dispersive spectroscopy (SEM-EDS) for the extent of densification and distribution of individual elements.

The preliminary investigation of HUP, HIP, and SPS showed that these sintering methods can be used to effectively consolidate powders of $\mathrm{Ag}^{0}$-functionalized silica aerogel into products of near-theoretical density. Also, removal of the organic moiety and adding 5.6 mass $\%$ of colloidal silica to $\mathrm{Ag}^{0}$ functionalized silica aerogel powders before processing resulted in denser products. Furthermore, the ram travel data for SPS indicated that rapid consolidation of powders with SPS can be performed at temperatures below $950{ }^{\circ} \mathrm{C}$. 


\section{CONTENTS}

SUMMARY iii

ABBREVIATIONS vi

1 INTRODUCTION 1

2

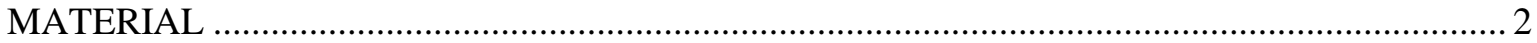

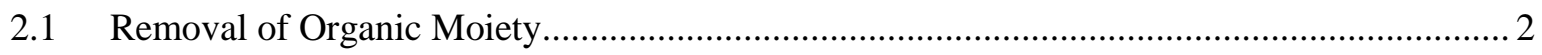

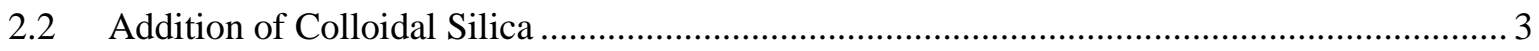

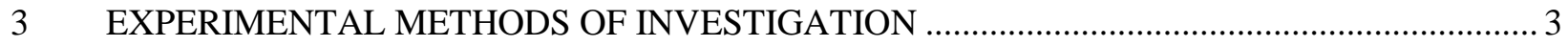

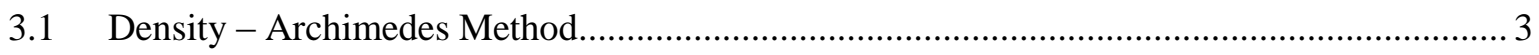

3.2 Density - Helium Pycnometry .............................................................................. 4

3.3 Scanning Electron Microscopy and Energy Dispersive Spectroscopy (SEM-EDS) .............. 4

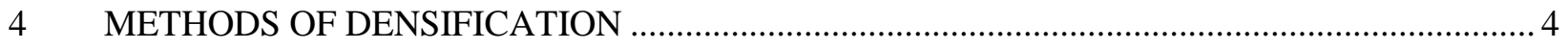

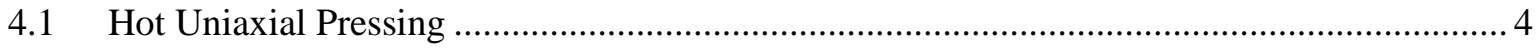

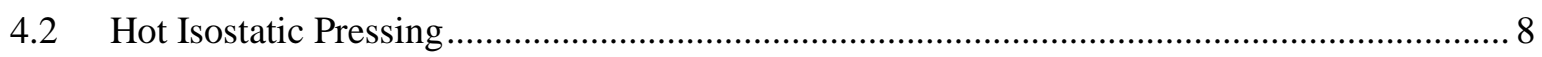

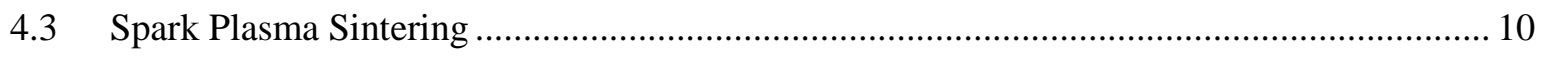

5 DISCUSSION

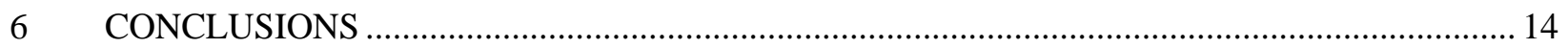

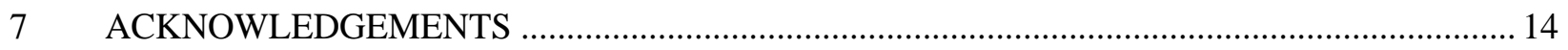

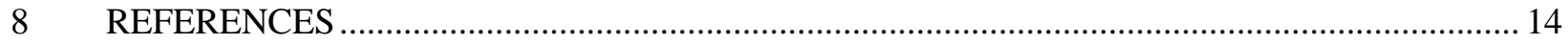

\section{FIGURES}

Figure 1. Results from the TGA analyses of $\mathrm{Ag}^{0}$-functionalized aerogel and iodine-loaded $\mathrm{Ag}^{0}$ functionalized silica aerogel for samples that were processed once and twice. The

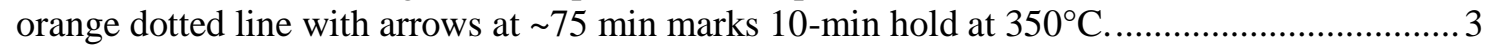

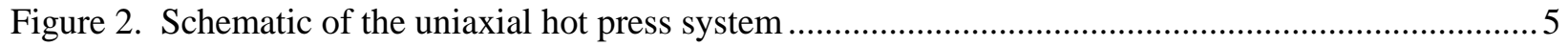

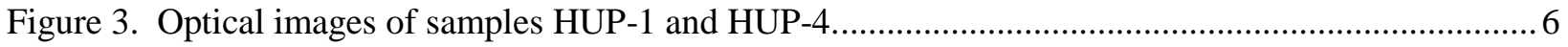

Figure 4. Apparent density and open porosity of HUP samples vs. increased concentration of added colloidal silica.

Figure 5. Backscattered electron images of partially fused silica grains for sample HUP-1. Cavities are the regions highlighted in red; nanoparticles and $\mu \mathrm{m}$-sized nodules of $\mathrm{Ag}$ show as bright white spots.

Figure 6. Backscattered electron image of fused silica grains for sample HUP-4. Nanoparticles and $\mu \mathrm{m}$-sized nodules of $\mathrm{Ag}$ are bright white spots.

Figure 7. Backscattered electron image of fused silica grains for sample HUP-6. Elemental analysis of areas $1-6$ is summarized in Table 1. 
Figure 8. Elemental dot map of the silica grain interface for sample HUP-4. 8

Figure 9. Photograph of tantalum envelope for HIPing of powders; A and B show the top of the envelope (top and bottom view of the circular dimple, respectively), and $\mathrm{C}$ shows the bottom of the envelope.

Figure 10. Processing conditions for HIPing.

Figure 11. Backscattered electron images of fused silica grains for sample HIP-2; image B shows Ta residue from the foil envelope.

Figure 12. Backscattered electron images of fused silica grains for sample HIP-3; image A shows areas 1-7 for elemental analysis, image $\mathrm{B}$ details $\mathrm{Ag}^{0}$ nanoparticles in a small region of area 4 of image A.

Figure 13. Apparent density and open porosity of SPS samples vs. increased concentration of added colloidal silica. Red-filled circle and blue-filled square represent sample SPS-1. Error bars were too small to be displayed....

Figure 14. Backscattered electron images of fused silica grains for sample SPS-3; image B details a small square area highlighted in image A and shows regions 1-4 used for elemental analysis

Figure 15. Backscattered electron images of fused silica grains for all SPS samples; white spots are nanoparticles and $\mu \mathrm{m}$-sized nodules of silver......

Figure 16. Pressure and ram displacement as functions of temperature for SPS samples.

\section{TABLES}

Table 1. Concentrations of $\mathrm{Ag}, \mathrm{S}, \mathrm{Si}$, and $\mathrm{O}$ in mass $\%$ for areas from Figure 7

(HUP-6, areas 1-6).

Table 2. Samples for HIPing

Table 3. Concentrations of $\mathrm{Ag}, \mathrm{S}, \mathrm{Si}$, and $\mathrm{O}$ in mass\% for areas from Figure 12

(HIP-3, areas 1-7).

Table 4. Concentrations of $\mathrm{Ag}, \mathrm{S}, \mathrm{Si}$, and $\mathrm{O}$ in mass $\%$ for areas from Figure 14B

(SPS-3, areas 1-4). 


\section{ABBREVIATIONS}

DC Direct current

DIW deionized water

HIP hot isostatic pressing

HUP hot uniaxial pressing

ICP-AES inductively coupled plasma-atomic emission spectroscopy

SEM-EDS scanning electron microscopy-energy dispersive spectroscopy

SPS spark plasma sintering

TGA-DSC Thermogravimetric analysis and differential scanning calorimetry 


\section{INTRODUCTION}

The revival of reprocessing of used nuclear fuel is being considered in the United States to recover valuable fissile constituents and isotopes, and to reduce the volume of highly radioactive material for storage or disposal. However, this process also generates a variety of waste streams, including volatile radionuclides, such as tritium $\left({ }^{3} \mathrm{H}\right)$, carbon-14 $\left({ }^{14} \mathrm{C}\right)$, krypton-85 $\left({ }^{85} \mathrm{Kr}\right)$, and iodine-129 $\left({ }^{129} \mathrm{I}\right)$, which are released into off-gas streams within the plant and must be captured to meet regulatory limits for their release (Jubin et al 2012). Of these radionuclides, ${ }^{129}$ I poses the greatest health risk as it has a half-life of $1.6 \times 10^{7}$ years and is highly mobile in the environment. It is therefore desirable to develop a material that can effectively capture ${ }^{129} \mathrm{I}$ and then be directly converted into a durable waste form for interim storage or long-term disposal.

A material that is capable of efficient capture and sequestration of radioiodine is $\mathrm{Ag}^{0}$-functionalized silica aerogel (Matyáš et al. 2011 and 2012). This novel sorbent has a high affinity for iodine at the low concentrations expected in the off-gas streams and a high sorption capacity, and, after loading with iodine, can be potentially consolidated into a dense, high-iodine-loaded and leach-resistant $\mathrm{SiO}_{2}$-based waste form. Previously, it was demonstrated to have a maximum sorption capacity for $\mathrm{I}_{2}$ of 48 mass $\%$ (Strachan et al. 2011), decontamination factors in excess of 10000 (99.99\% of iodine removed) (Soelberg et al. 2012), good sorption performance after long-term exposure to dry air (simulating process idling) (Bruffey et al. 2012, Matyáš et al. 2012a), and retention of more than $92 \%$ of iodine in densified waste form after iodine-loaded aerogel was processed at $1200{ }^{\circ} \mathrm{C}$ for $30 \mathrm{~min}$ under low pressure (Matyáš et al. 2011). It is expected that iodine retention can be further improved by optimizing the sintering process through a simultaneous application of fast heating rates to temperatures above $1000{ }^{\circ} \mathrm{C}$ and pressures up to $210 \mathrm{MPa}$. Under these conditions a fast sintering of intimately contacted particles occurs, which will likely result in higher iodine retention.

The main purpose of this report is to provide a preliminary evaluation of commercially available technologies with which iodine-loaded $\mathrm{Ag}^{0}$-functionalized silica aerogel can be rapidly consolidated to a durable $\mathrm{SiO}_{2}$-based waste form. In this study, $\mathrm{Ag}^{0}$-functionalized silica aerogel powders were consolidated/sintered with three methods: 1) hot uniaxial pressing (HUP), which involves heating samples in an open-air furnace while simultaneously applying pressure along a single axis, 2) hot isostatic pressing (HIP), which involves heating samples in enclosed metal envelopes in a sealed furnace while simultaneously applying equal pressure from all directions, and 3) spark plasma sintering (SPS), which involves heating samples via internal resistance heating under high applied voltage while simultaneously applying pressure along a single axis. Since there are no studies reported in literature on the sintering of aerogels with HIP and SPS the preliminary experiments were performed with powders containing no iodine and were focused on investigating feasibility of these methods to produce a fully dense product. This was also a conservative approach to avoid potential damage to vendor equipment, if significant quantities of iodine were have released during consolidation.

Another purpose of this report was to investigate the effect of organic moiety in the $\mathrm{Ag}^{0}$-functionalized silica aerogel and colloidal silica on densification process. There is a potential that the decomposition of organic moiety that is used to bind the $\mathrm{Ag}$ to the solid substrate by providing $\mathrm{Si}$ - and $\mathrm{Ag}$-active links could impede the sintering of $\mathrm{Ag}^{0}$-functionalized silica aerogel to near theoretical density. Furthermore, high silver loadings in silica aerogel might prevent fast and efficient consolidation to the waste form because the available silica might be insufficient for encapsulation of iodine in the form of AgI particles. Therefore, thermogravimetric analysis coupled with differential scanning calorimetry (TGA-DSC) was used to identify an optimum heat-treatment regime for removal of the organic moiety from the pores surfaces of iodine-loaded $\mathrm{Ag}^{0}$-functionalized silica aerogel, and colloidal silica was added to a few samples to investigate its impact on the extent of densification. 


\section{MATERIAL}

For this study, a batch of $\mathrm{Ag}^{0}$-functionalized silica aerogel was synthesized with a previously developed procedure (Matyáš et al. 2011). The granules were hand-ground for 2 min with an agate mortar and pestle and analyzed for the concentrations of $\mathrm{Ag}, \mathrm{S}$, and $\mathrm{Si}$ with inductively coupled plasma atomic emission spectroscopy (ICP-AES). The sample contained 25.9 mass\% Ag, 6.4 mass\% S, and 26.3 mass\% Si (56.3 mass\% $\mathrm{SiO}_{2}$ ). The remaining 11.4 mass\% was assigned to the functional organic moiety. This moiety can be removed during a pretreatment step. Therefore, normalized concentrations of Ag, S, and $\mathrm{SiO}_{2}$ were 29.3, 7.2, and 63.5 mass\%, respectively. With these concentrations and the densities of $\mathrm{Ag}$ $\left(\rho_{\mathrm{Ag}}=10.49 \times 10^{3} \mathrm{~kg} / \mathrm{m}^{3}\right), \mathrm{S}\left(\rho_{\mathrm{S}}=2.07 \times 10^{3} \mathrm{~kg} / \mathrm{m}^{3}\right)$, and $\mathrm{SiO}_{2}$ (density of fully consolidated silica aerogel $\rho_{\mathrm{SiO} 2}=2.2 \times 10^{3} \mathrm{~kg} / \mathrm{m}^{3}$, (Matyáš et. al. 2012) a theoretical density of $2.85 \times 10^{3} \mathrm{~kg} / \mathrm{m}^{3}$ was calculated for the functionalized aerogel. Adding 5.6, 10.5, and 15 mass $\%$ of colloidal silica decreased the theoretical density to $2.80,2.76,2.73 \times 10^{3} \mathrm{~kg} / \mathrm{m}^{3}$, respectively.

Powders of Ag-functionalized silica aerogels, with or without organic moiety and with or without sintering additive (up to 15 mass $\%$ of colloidal silica) were prepared for sintering with HUP, HIP, and SPS.

\subsection{Removal of Organic Moiety}

The small granular samples $(20-30 \mathrm{mg})$ of $\mathrm{Ag}^{0}$-functionalized silica aerogel with and without iodine were loaded into a Pt crucible, heated at $5{ }^{\circ} \mathrm{C} / \mathrm{min}$ from room temperature to $350{ }^{\circ} \mathrm{C}$ under flowing air $\left(25 \mathrm{~mL} / \mathrm{min}\right.$ at $\left.25^{\circ} \mathrm{C}\right)$, and held at this temperature for $2 \mathrm{~h}$ before being air quenched. This TGA-DSC (TA Instruments SDT Q-600, TA Instruments, New Castle, DE) heat treatment was done twice on the same iodine-loaded sample; i.e., the already processed sample [Iodine-loaded (I)] was left in the furnace exposed to open air overnight before being heat treated again under the same conditions [Iodine-loaded (II)], to determine the removal efficiency of the organic moiety and to investigate the effect of pretreatment on the retention of iodine.

Figure 1 shows TGA curves for $\mathrm{Ag}^{0}$-functionalized silica aerogel and iodine-loaded $\mathrm{Ag}^{0}$-functionalized silica aerogel after the first [Iodine-loaded (I)] and second [Iodine-loaded (II)] heat treatment. The $\mathrm{Ag}^{0}$-functionalized silica aerogel exhibited two distinct mass losses. First, 1.7 mass $\%$ of physisorbed water (residual water from the synthesis of $\mathrm{Ag}^{0}$-functionalized silica aerogel) was lost at temperatures below $110{ }^{\circ} \mathrm{C}$, and then there was a 10.6 mass $\%$ loss from the decomposition of the organic moiety during heating to $350{ }^{\circ} \mathrm{C}$ and $10 \mathrm{~min}$ hold. For the same time/temperature range, the iodine-loaded aerogels [Iodine-loaded (I) and (II)] showed losses of 2.2 and 4.4 mass\% of physisorbed water, respectively, and 8.8 and 1.2 mass $\%$ of organic moiety, respectively. The presence of twice as much physically adsorbed water in the Iodine-loaded (II) sample provided evidence of efficient removal of the organic moiety by heating to $350^{\circ} \mathrm{C}$ and 10 min hold: a part of the aerogel surface was no longer hydrophobic. Also, the TGA results indicated that the ramp heating to $350{ }^{\circ} \mathrm{C}$ and $10 \mathrm{~min}$ dwell time did not remove captured iodine and can be therefore used as a pretreatment step before high-temperature densification. 


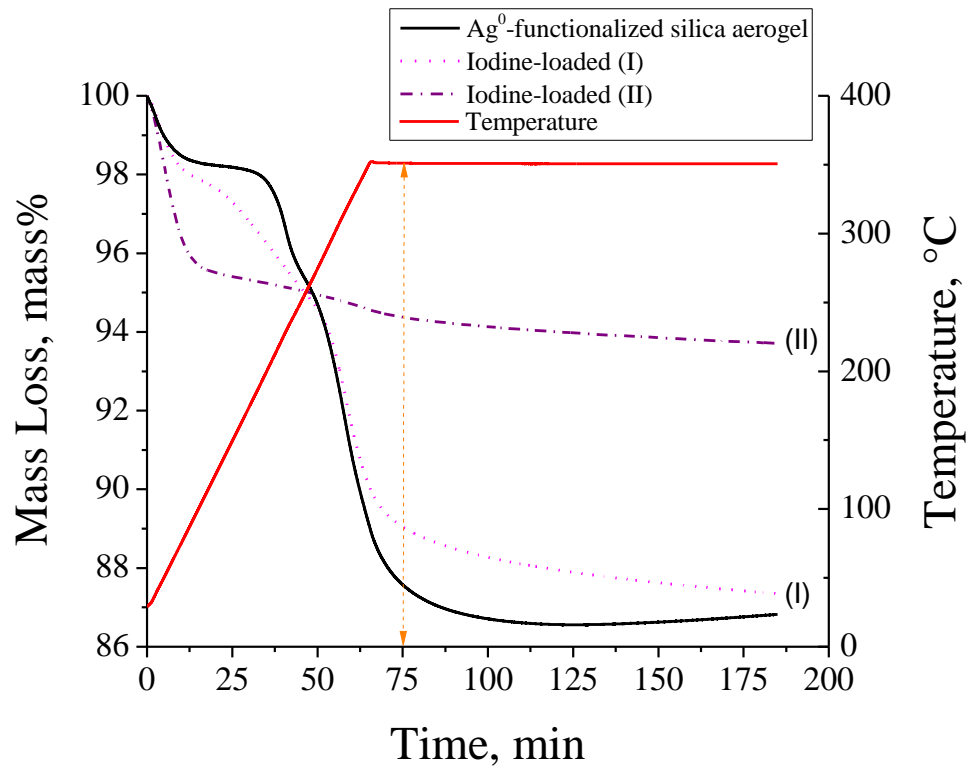

Figure 1. Results from the TGA analyses of $\mathrm{Ag}^{0}$-functionalized aerogel (black line) and iodine-loaded $\mathrm{Ag}^{0}$-functionalized silica aerogel for samples that were processed once [pink dot line, Iodine-loaded (I)] and twice [purple dash-dot line, Iodine-loaded (II)]. The orange dotted line with arrows at $\sim 75$ min marks 10 -min hold at $350{ }^{\circ} \mathrm{C}$.

\subsection{Addition of Colloidal Silica}

Granules of $\mathrm{Ag}^{0}$-functionalized silica aerogel were hand ground for 2 min with an agate mortar and pestle before different amounts of colloidal silica solutions were added with pipettes. Powders with or without colloidal silica (Ludox ${ }^{\circledR}$ CL-X colloidal silica, 45 mass\% suspension in $\mathrm{H}_{2} \mathrm{O}$, Sigma Aldrich) were then heat treated in Pt crucibles according to a schedule determined from TGA-DSC (powders were ramped from room temperature to $350{ }^{\circ} \mathrm{C}$ at a rate of $5{ }^{\circ} \mathrm{C} / \mathrm{min}$, held at $350{ }^{\circ} \mathrm{C}$ for $10 \mathrm{~min}$, and air quenched) to remove the organic moiety and water, and to prepare samples containing 5.6, 10.5, and 15 mass\% extra silica.

\section{EXPERIMENTAL METHODS OF INVESTIGATION}

\subsection{Density - Archimedes Method}

The samples of $\mathrm{Ag}^{0}$-functionalized silica aerogel that were consolidated with HUP and SPS were weighed on an analytical balance (Sartorius model A200S, Brinkmann Instruments Co., Westbury, New York) to an accuracy of $0.1 \mathrm{mg}\left(m_{d r y}\right)$ and then suspended in deionized water (DIW) for $2 \mathrm{~h}$ under in-house vacuum $(-91.4 \mathrm{kPa})$ to allow the DIW to penetrate any open and surface-connected porosity. Next, the samples were weighed both suspended in DIW $\left(m_{\text {sus }}\right)$ and in air saturated with DIW $\left(m_{\text {sat }}\right)$. The apparent densities of samples were calculated according to Equation (1):

$$
\rho_{\text {sample }}=\frac{m_{d r y} \rho_{D I W}}{\left(m_{\text {sat }}-m_{\text {sus }}\right)}
$$

where $m_{i}$ is the mass in the $i$-th configuration ( $i=$ dry, saturated and suspended, respectively), $\rho_{D I W}$ is the density of DIW at the temperature of the test $\left(0.9981 \times 10^{3} \mathrm{~kg} / \mathrm{m}^{3}\right.$ at $\left.20.5^{\circ} \mathrm{C}\right)($ ASTM C693-93). The open porosity of samples was calculated according to Equation (2):

$$
\% \text { OpenPorosity }=\frac{\left(m_{\text {sat }}-m_{d r y}\right)}{\left(m_{\text {sat }}-m_{\text {sus }}\right)} \times 100
$$


A sample of aluminum with a density $2.7000 \times 10^{3} \mathrm{~kg} / \mathrm{m}^{3}$ and zero open porosity was run with the samples to validate obtained densities and open porosities. The measured density, $2.6946 \times 10^{3} \mathrm{~kg} / \mathrm{m}^{3}$, and open porosity, $0.26 \%$, were in good agreement with values for aluminum metal.

\subsection{Density - Helium Pycnometry}

A helium pycnometer (AccuPyc II 1340, Micromeritics Inc., Norcross, GA) was used to determine the apparent volume (the volume of the solid material excluding surface-connected porosity, but including closed pores) of two HIP samples by measuring the pressure change of helium (99.995\%) in a calibrated volume. The apparent density of samples was calculated from the known sample masses. The pycnometer was operated with a $1 \mathrm{~mL}$ sample cup. The volume of the cup was calibrated with a $0.7182 \pm 0.0001 \mathrm{~mL}$ tungsten cylinder. The calibration was checked with a sample of aluminum of known density, $2.7000 \times 10^{3} \mathrm{~kg} / \mathrm{m}^{3}$, at $20^{\circ} \mathrm{C}$. The measured density of $2.7023 \pm 0.0152 \times 10^{3} \mathrm{~kg} / \mathrm{m}^{3}$ was in good agreement with known value.

The samples were weighed on an analytical balance to the nearest $0.1 \mathrm{mg}$. Sample masses were 0.2145 and $0.1672 \mathrm{~g}$ for samples HIP-2 and HIP-3, respectively. The cup with samples was purged (cell filling/expulsion of helium) 10 times to remove air and moisture from the sample and the inside of the chamber. This was followed by 10 measurement cycles of the sample volume for density determination, the results of which were averaged.

\subsection{Scanning Electron Microscopy and Energy Dispersive Spectroscopy (SEM-EDS)}

The samples were characterized with a SEM JSM-7001F/TTLS (JEOL Ltd., Tokyo, Japan) equipped with a field emission gun and capable of examining specimens under variable pressure conditions, allowing them to be evaluated without a conductive coating. The SEM was operated in low vacuum mode at an accelerating voltage of $15 \mathrm{kV}$ to minimize beam penetration; with low electron-beam penetration depth, elemental analyses and imaging resolution were improved. Backscattered electron imaging was used to show changes in atomic number $(\mathrm{Z})$, maximizing the detection of differences in chemistry. Polished cross-sections or chipped surfaces were examined for the extent of densification of $\mathrm{Ag}^{0}$-functionalized silica aerogel powders, the amount of open porosity, and the character of grain boundaries. Elemental spot analyses were collected for different areas. An elemental dot map was also collected to reveal the distribution of individual elements throughout densified sample.

\section{METHODS OF DENSIFICATION}

Three methods of consolidation were used in this study: 1) hot uniaxial pressing, 2) hot isostatic pressing, and 3) spark plasma sintering. In this section, each method is briefly described and the results discussed with regard to the extent of powder densification.

\subsection{Hot Uniaxial Pressing}

Figure 2 shows a schematic of the uniaxial hot press system that was designed and fabricated at Pacific Northwest National Laboratory (PNNL) (Matyáš et al. 2012). In this system, the unidirectional pressure and heat from external heating elements are applied simultaneously to enhance the densification rate of the samples.

Powdered 0.2-gsamples of $\mathrm{Ag}^{0}$-functionalized silica aerogel were each loaded into an alumina crucible $(9.55 \mathrm{~mm}$ OD $\times 6.42 \mathrm{~mm}$ ID $\times 19.10 \mathrm{~mm}$ tall $)$ and subsequently compressed with $6 \mathrm{~kg}$ weight on the plunger rod. This crucible was secured with a Pt wire spring to the alumina push rod, which was mounted to the Duramaster rod cylinder (Greenco Mfg. Corporation, Tampa, FL) outside of the furnace. The rod cylinder was pressurized with air, extending a push rod and compressing the sample in the crucible with $29 \mathrm{MPa}$ pressure against a mullite block inside the furnace that was preheated to $1200{ }^{\circ} \mathrm{C}$. After $30 \mathrm{~min}$, 
the rod was retracted from the furnace, the crucible was removed, and the sample air quenched on an alumina plate.

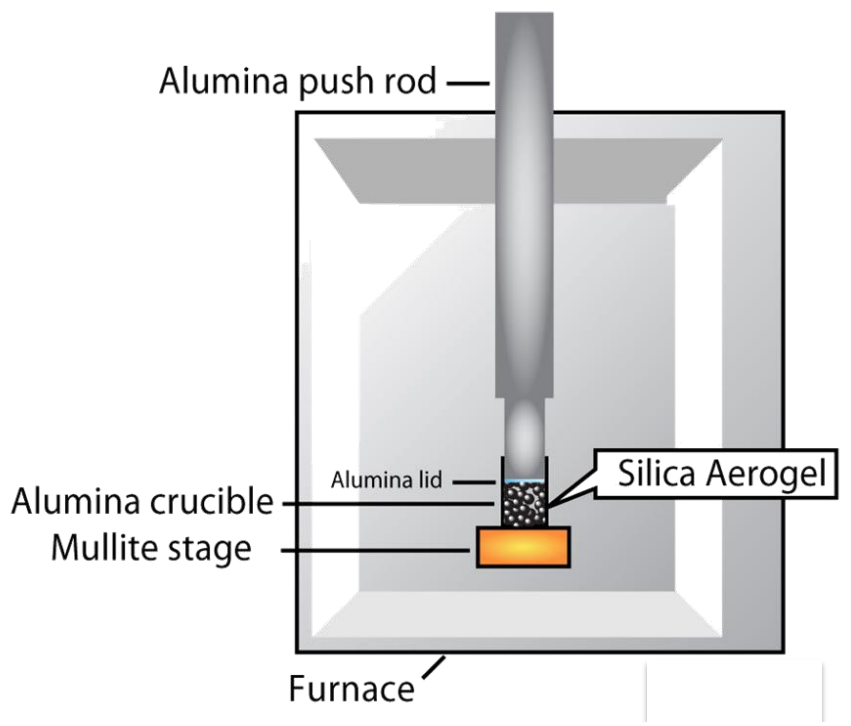

Figure 2. Schematic of the uniaxial hot press system

Four samples of $\mathrm{Ag}^{0}$-functionalized silica aerogel were processed with HUP. Each sample had the organic moiety removed and contained a different amount of added colloidal silica. Samples HUP-1, $-4,-5$, and -6 contained $0,5.6,10.5$ and 15 mass $\%$ of extra silica, respectively.

Figure 3 shows optical images of the polished surfaces of the partially sintered HUP-1 and densified HUP-4 samples. Figure 4 shows the change of apparent and theoretical density, and open porosity for HUP samples with increased concentration of colloidal silica. The addition of 5.6 mass\% silica (HUP-2) increased the density of the product from $2.49 \pm 0.02 \times 10^{3}$ to $2.72 \pm 0.02 \times 10^{3} \mathrm{~kg} / \mathrm{m}^{3}$ but, more importantly, decreased open porosity from 15.4 to $3.8 \%$. This open porosity did not change with addition of 10.5 mass $\%$ of silica, but decreased to $2.0 \%$ when 15 mass $\%$ silica was added (HUP-6). Powders in samples HUP-4, -5 , and -6 were consolidated to $97-99 \%$ theoretical density. The drop in density observed for HUP-6 was caused by a diluting effect, i.e., the concentration of highly dense silver is diluted by less-dense silica that did not fill open pores. There was no evidence of bloating, which can generate porosity. Figure 5 shows SEM backscattered images of partially sintered sample HUP-1, visualizing cavities at grain boundaries and individual silica grains containing uniformly dispersed nanoparticles of $\mathrm{Ag}$ and $\mathrm{Ag}_{2} \mathrm{~S}$. In addition, a few $\mu$ m-sized nodules of $\mathrm{Ag}$ and $\mathrm{Ag}_{2} \mathrm{~S}$ formed at interfaces between grains. These phases were confirmed with an X-ray diffraction. Figure 6 shows a detailed SEM backscattered image of fused silica grains for the consolidated HUP-4 sample; the lighter the area of silica grains, the higher the concentration of silver nanoparticles. Figure 7 shows an SEM backscattered image of a chipped piece of the sample HUP-6, including areas that were analyzed with EDS for concentration of $\mathrm{Ag}, \mathrm{S}, \mathrm{Si}$, and $\mathrm{O}$. Table 1 summarizes the concentrations of these elements. Concentration of $\mathrm{Ag}$ varied from $\sim 0.3$ mass \% (dark-grey area) and $>27$ mass $\%$ (medium-grey area) to $>42.5$ mass $\%$ (lightgrey area). As much as 12 mass $\%$ of $\mathrm{S}$ from the thiolation process was present in the light-grey areas. Figure 8 shows an elemental dot map detailing the distribution of $\mathrm{Ag}, \mathrm{S}, \mathrm{Si}$, and $\mathrm{O}$ over a small area of the sample HUP-4. 

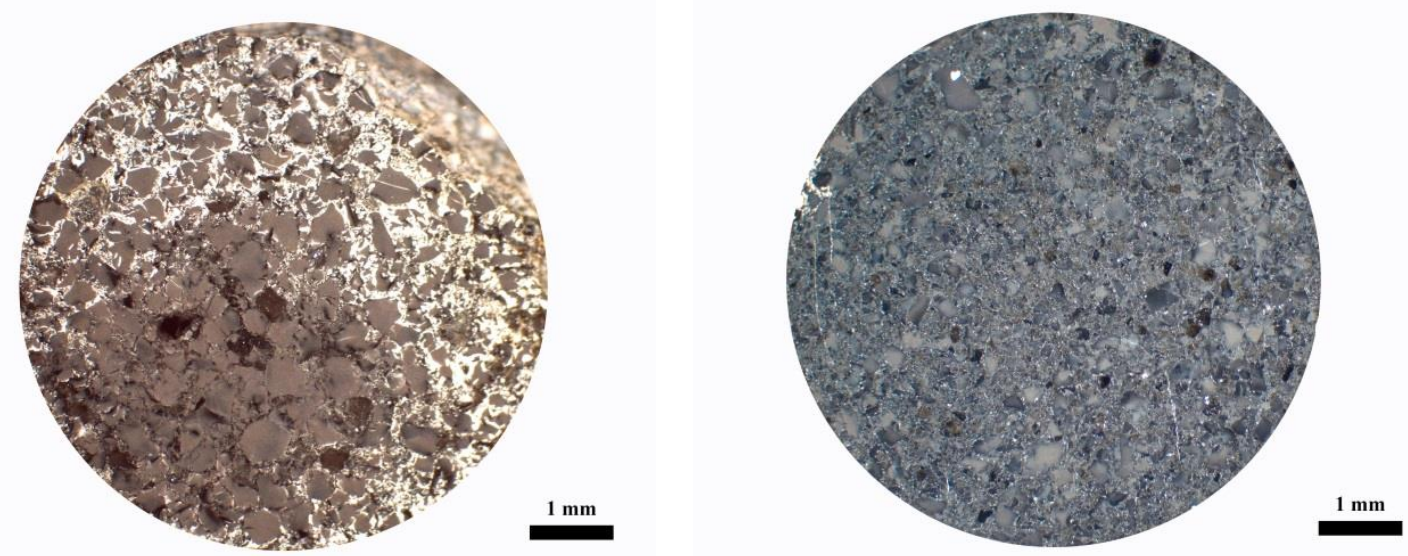

Figure 3. Optical images of samples HUP-1 and HUP-4 $\left(1200^{\circ} \mathrm{C}\right.$ for 30 min under $\left.29 \mathrm{MPa}\right)$.

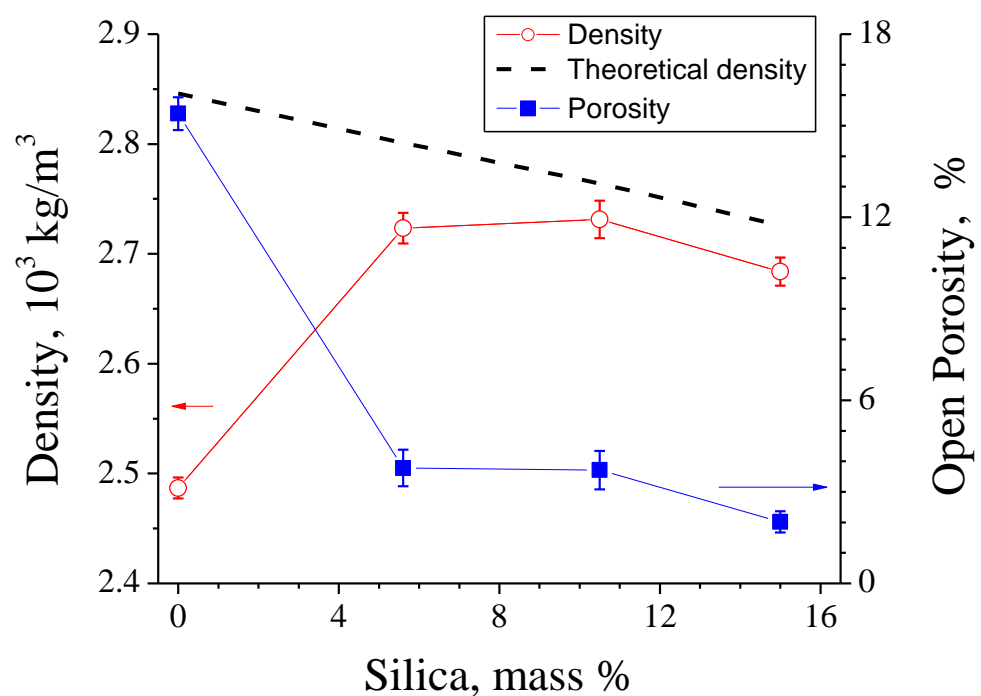

Figure 4. Apparent density and open porosity of HUP samples vs. increased concentration of added colloidal silica.
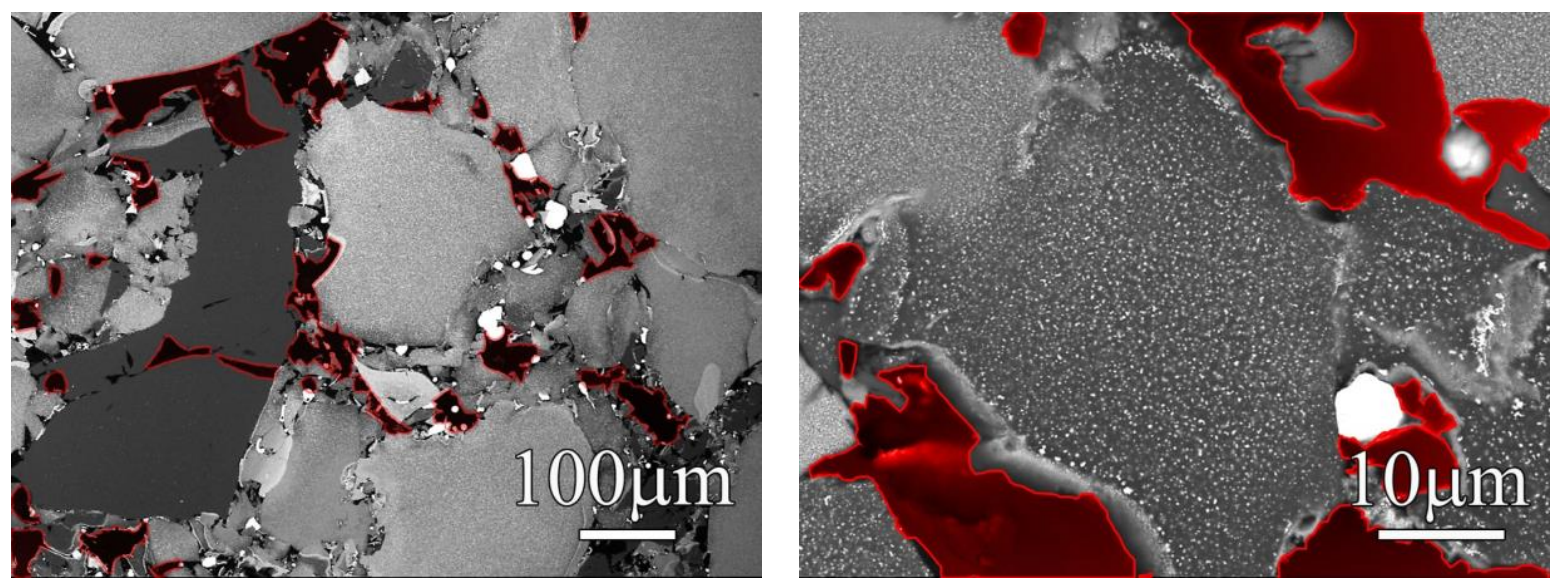

Figure 5. Backscattered electron images of partially fused silica grains for sample HUP-1. Pores are the regions highlighted in red; nanoparticles and $\mu \mathrm{m}$-sized nodules of $\mathrm{Ag}$ show as bright white spots. 


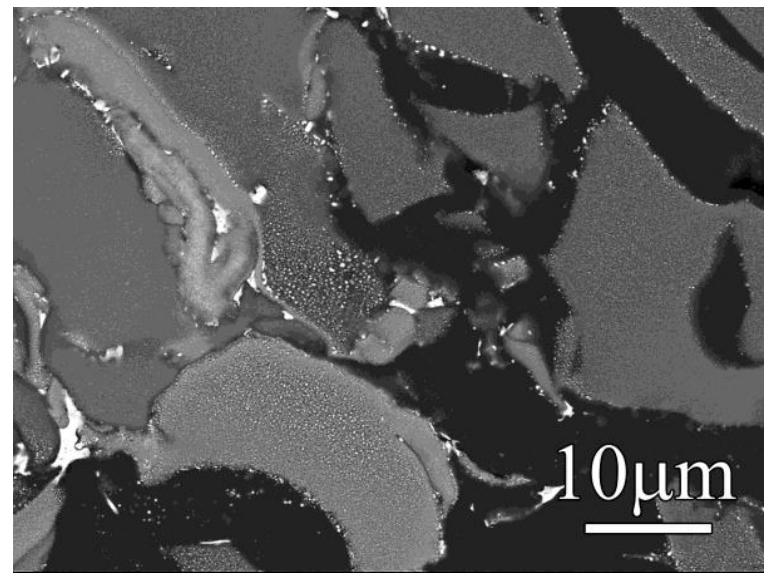

Figure 6. Backscattered electron image of fused silica grains for sample HUP-4. Nanoparticles and $\mu \mathrm{m}$-sized nodules of Ag are bright white spots.

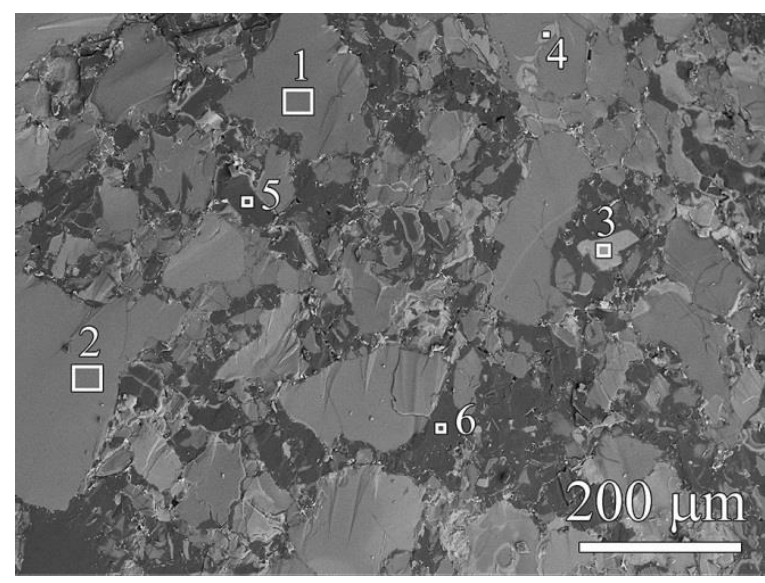

Figure 7. Backscattered electron image of fused silica grains for sample HUP-6. Elemental analysis of areas 1-6 is summarized in Table 1.

Table 1. Concentrations of Ag, S, Si, and O in mass\% for areas from Figure 7 (HUP-6, areas 1-6).

\begin{tabular}{|c|r|r|r|r|}
\hline \multirow{2}{*}{ Area } & \multicolumn{4}{|c|}{ Concentration, mass\% } \\
\cline { 2 - 5 } & \multicolumn{1}{c|}{$\mathrm{Ag}$} & \multicolumn{1}{c|}{$\mathrm{S}$} & \multicolumn{1}{c|}{$\mathrm{Si}$} & \multicolumn{1}{c|}{$\mathrm{O}$} \\
\hline 1 & 27.54 & 4.88 & 55.19 & 12.39 \\
\hline 2 & 31.75 & 7.96 & 51.11 & 9.18 \\
\hline 3 & 42.95 & 12.18 & 38.92 & 5.95 \\
\hline 4 & 45.10 & 12.56 & 36.72 & 5.62 \\
\hline 5 & 0.27 & 1.84 & 71.74 & 26.15 \\
\hline 6 & 0.48 & 2.03 & 74.60 & 22.89 \\
\hline
\end{tabular}




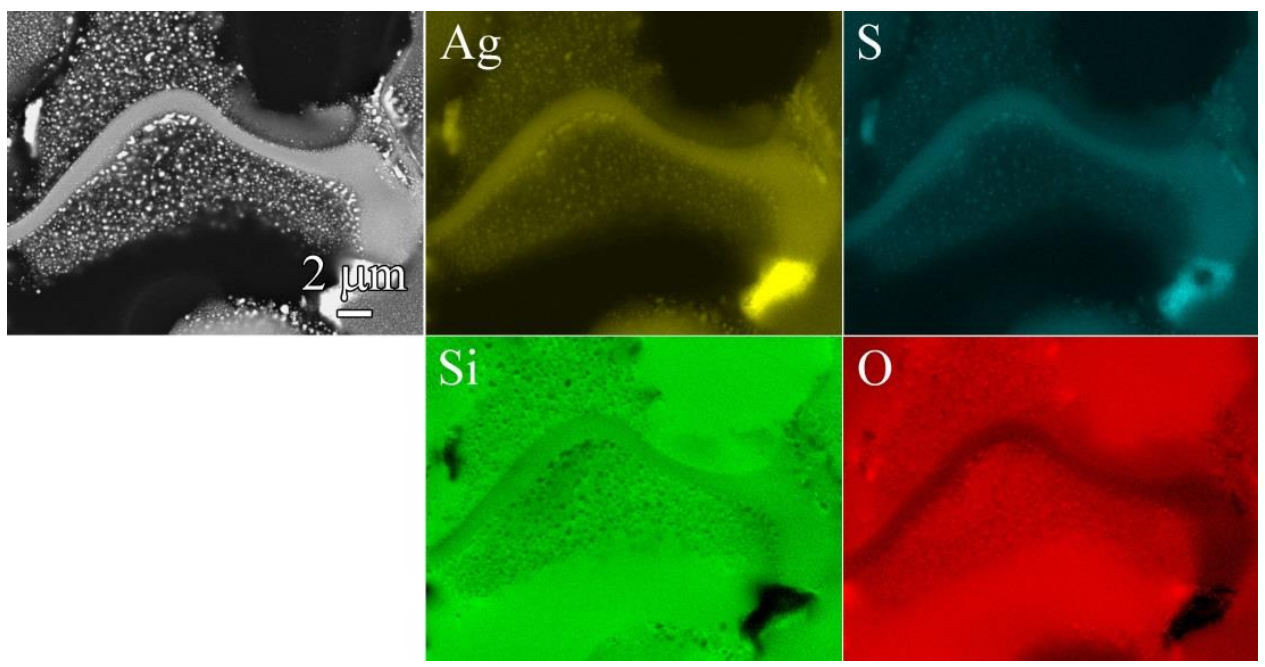

Figure 8. Elemental dot map of the silica grain interface for sample HUP-4.

\subsection{Hot Isostatic Pressing}

Hot isostatic pressing is a thermal treatment method in which the pressure and temperature are applied equally on all sides (isostatically) of the sample with a compressed and heated inert gas such as argon. In general, the powder is consolidated to near theoretical density in an evacuated metal container that is consolidated along with the contained powder as the pressure and temperature are increased. The pressures can be as high as $310 \mathrm{MPa}$ and the temperatures as high as $2000{ }^{\circ} \mathrm{C}$ (Alcoa Howmet Hot Isostatic Pressing).

Table 2 shows four 0.4-g samples of $\mathrm{Ag}^{0}$-functionalized silica aerogel powders prepared for HIP: 1) powder containing organic moiety (HIP-1), 2) powder that had organic moiety removed (HIP-2), 3) powder that had organic moiety removed but contained 5.6 mass $\%$ of added colloidal silica (HIP-3), and 4) powder that had organic moiety removed but contained 10.5 mass $\%$ of added colloidal silica (HIP4). Figure 9 details the design of the tantalum envelope made from 0.016-mm foil. Each powdery sample was lightly compacted in the $15-\mathrm{mm}$ circular dimple of the circular sheet (38 $\mathrm{mm}$ in diameter) and encapsulated by a circular flat sheet of the same diameter. These envelopes were then degassed at approximately $0.01 \mathrm{~Pa}$, sealed with electron-beam welding and hot isostatic pressed at American Isostatic Pressing (Columbus, Ohio) in a research-scale HIP, which had carbon heating elements and used argon gas to pressurize the chamber. All four envelopes were processed at the same time and were heated at $20{ }^{\circ} \mathrm{C} / \mathrm{min}$ from room temperature to $1200{ }^{\circ} \mathrm{C}$ with a 30 -min hold and consolidated under $207 \mathrm{MPa}$. Figure 10 shows the temperature and pressure profiles for the run.

Table 2. Samples for HIPing

\begin{tabular}{|c|c|c|}
\hline Sample ID & Organic moiety & Colloidal silica, mass\% \\
\hline HIP-1 & present & 0 \\
\hline HIP-2 & removed & 0 \\
\hline HIP-3 & removed & 5.6 \\
\hline HIP-4 & removed & 10.5 \\
\hline
\end{tabular}




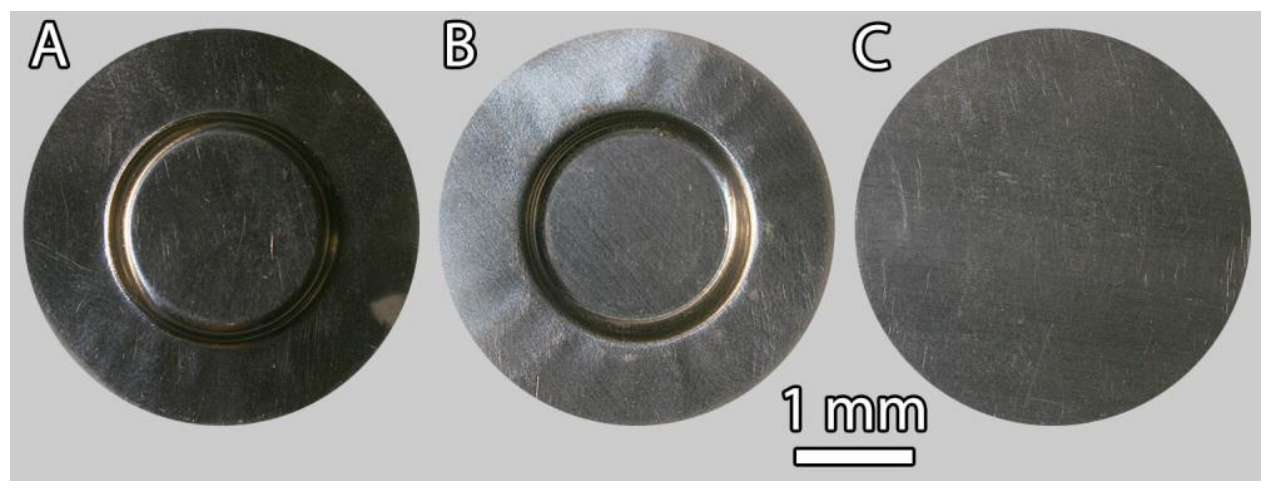

Figure 9. Photograph of tantalum envelope for HIP processing of powders; A and B show the top of the envelope (top and bottom view of the circular dimple, respectively), and $\mathrm{C}$ shows the bottom of the envelope.

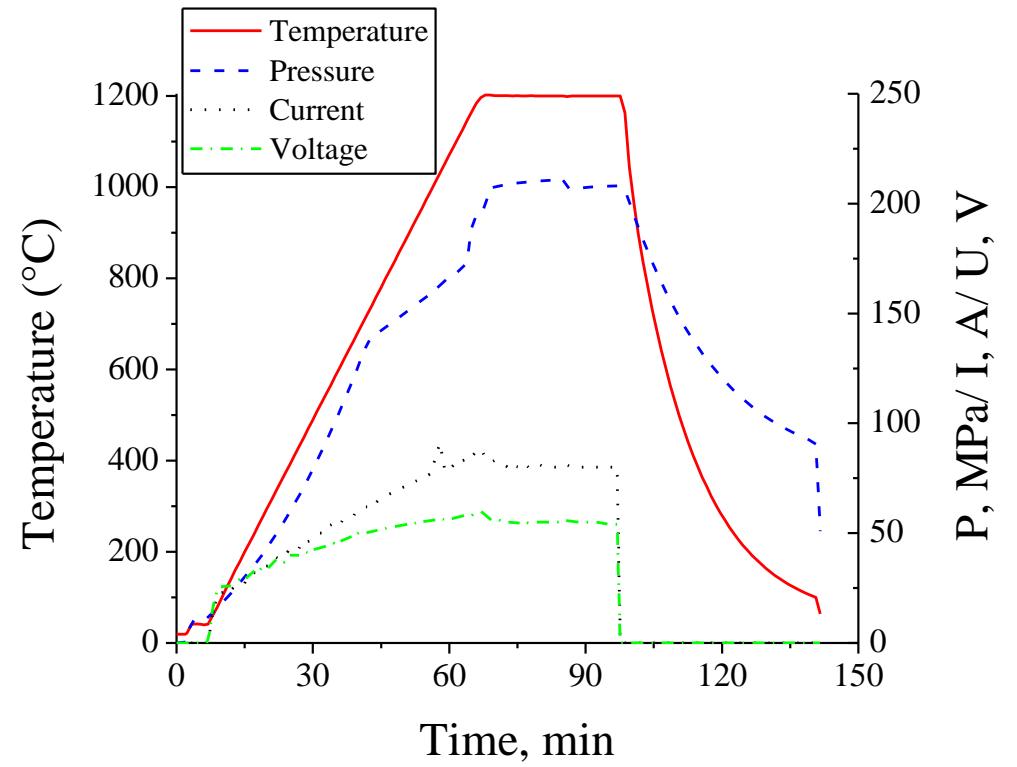

Figure 10. Processing conditions for HIPing (temperature, pressure, current, and voltage as functions of time).

Samples HIP-1 and HIP-4 failed during processing. A plastic deformation of envelopes early in the process resulted in cracked metal foil that prevented consolidation of powders. The powders from the other two envelopes, HIP-2 and -3 , were consolidated into plates $\sim 0.3 \mathrm{~mm}$ thick and $15 \mathrm{~mm}$ diameter. These plates were strongly attached to the walls of envelope and therefore had to be broken into smaller pieces during sample recovery. Recovered pieces were too small for the measurement of density and open porosity with the Archimedes method, but the measurement of apparent density with the He gas pycnometer could be made. Densities of HIP-2 and HIP-3 were $3.03 \pm 0.04 \times 10^{3}$ and $2.83 \pm 0.03 \times 10^{3}$ $\mathrm{kg} / \mathrm{m}^{3}$, respectively. The high density of HIP-2 can be explained by contamination of the sample surface with Ta residue from the Ta foil envelope. Figure 11A and Figure 11B show backscattered electron images of pieces from sample HIP-2, with Figure 11B showing the detail of the Ta layer on the surface. Similar to HUP samples, HIP powders were composed of fused silica grains containing different concentrations of Ag nanoparticles. However, products that exhibited higher densities did not contain $\mu \mathrm{m}$-sized nodules of $\mathrm{Ag}$ and cavities at the grain boundaries, suggesting that HIPing produced neartheoretically dense plates. Table 3 summarizes the concentrations of $\mathrm{Ag}, \mathrm{S}, \mathrm{Si}$, and $\mathrm{O}$ in mass $\%$ for the areas in Figure 12. Concentration of $\mathrm{Ag}$ varied from $~ 0.3$ mass \% (dark-grey areas) and $>27$ mass $\%$ (medium-grey areas) to $>40$ mass\% (light-grey areas). 
A
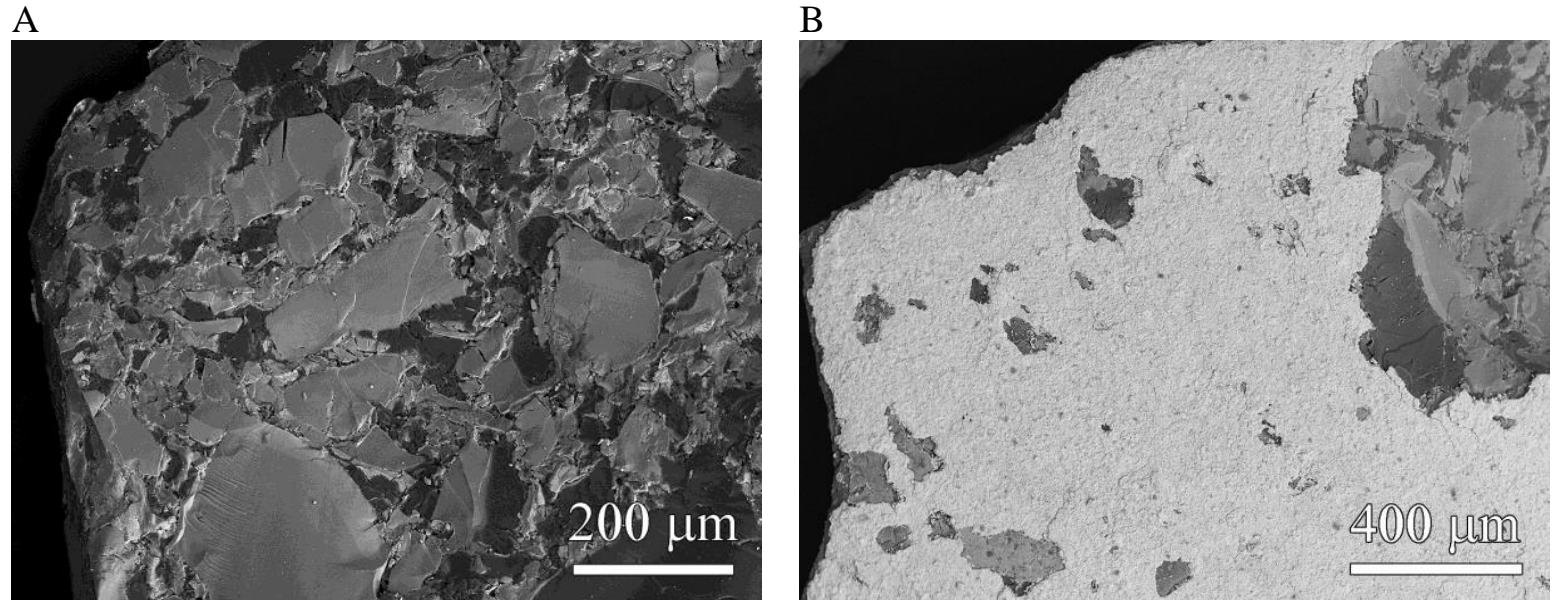

Figure 11. Backscattered electron images of fused silica grains for sample HIP-2; image B shows Ta residue (white layer) from the foil envelope.

A

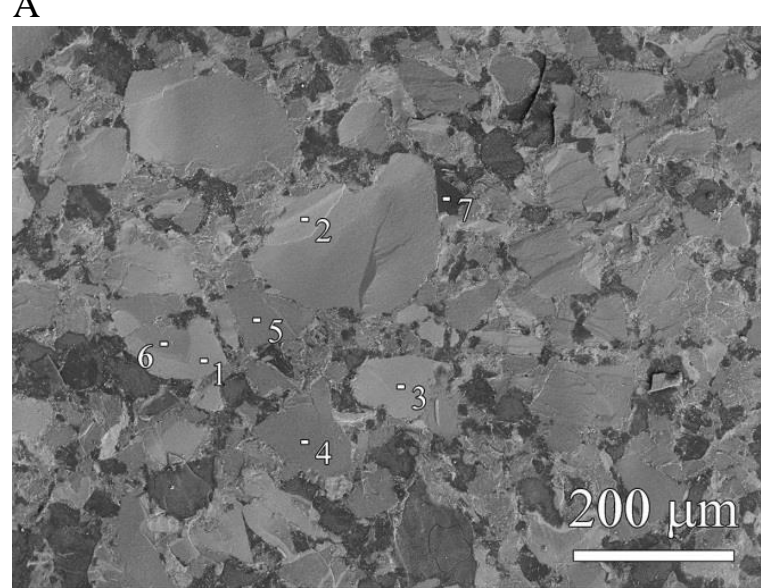

B

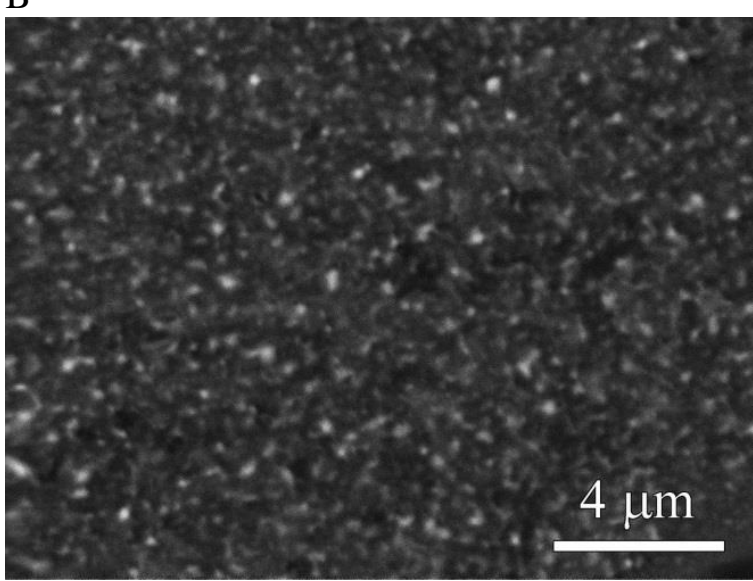

Figure 12. Backscattered electron images of fused silica grains for sample HIP-3; image A shows areas 1-7 for elemental analysis (summarized in Table 3), image $\mathrm{B}$ details $\mathrm{Ag}^{0}$ nanoparticles (white spots) in a small region of area 4 of image A.

Table 3. Concentrations of Ag, S, Si, and $\mathrm{O}$ in mass\% for areas from Figure 12 (HIP-3, areas 1-7).

\begin{tabular}{|c|c|c|c|c|}
\hline \multirow{2}{*}{ Area } & \multicolumn{4}{|c|}{ Concentration, mass\% } \\
\cline { 2 - 5 } & $\mathrm{Ag}$ & $\mathrm{S}$ & $\mathrm{Si}$ & $\mathrm{O}$ \\
\hline 1 & 40.39 & 11.35 & 39.70 & 8.56 \\
\hline 2 & 41.59 & 10.36 & 41.04 & 7.01 \\
\hline 3 & 41.54 & 11.38 & 39.16 & 7.93 \\
\hline 4 & 27.67 & 6.82 & 50.83 & 14.68 \\
\hline 5 & 28.83 & 7.45 & 49.74 & 13.97 \\
\hline 6 & 31.34 & 7.76 & 48.78 & 12.11 \\
\hline 7 & 0.35 & 0.40 & 70.76 & 28.49 \\
\hline
\end{tabular}

\subsection{Spark Plasma Sintering}

Spark plasma sintering is a rapid sintering method that utilizes a simultaneous application of uniaxial pressure and a pulsed direct current (DC) under low atmospheric pressure to consolidate powder materials. A high DC pulse that is applied across a die generates heating power that is dissipated at the 
contact points of powder particles. This homogeneous distribution of the heat at the microscopic scale results in heating rates as high as $1000{ }^{\circ} \mathrm{C} / \mathrm{min}$ (Zhang et al. 2013).

A total of four samples of $\mathrm{Ag}^{0}$-functionalized silica aerogel (identical to samples processed with HIP) were processed at California Nanotech Corporation (Cerritos, California) with a laboratory-scale sparkplasma instrument, Fuji Electronic Dr. Sinter Lab Series SPS-515S (Syntex Inc., Kanagawa, Japan). Samples SPS-1 and SPS-2 contained powders of as-prepared $\mathrm{Ag}^{0}$-functionalized silica aerogel with the organic moiety present or removed, respectively. The organic moiety was also removed from samples SPS-3 and SPS-4, but 5.3 mass\% and 9.5 mass $\%$ of colloidal silica was added to these samples, respectively, to improve the sintering process and increase the density of the product. Each sample was loaded into a 10-mm diameter high-strength graphite die and rapidly step-wise heated to $1200{ }^{\circ} \mathrm{C}$. Powders were heated under argon and $39 \mathrm{MPa}$ pressure from room temperature to $500{ }^{\circ} \mathrm{C}$ at $158{ }^{\circ} \mathrm{C} / \mathrm{min}$, from 500 to $1100{ }^{\circ} \mathrm{C}$ at $300{ }^{\circ} \mathrm{C} / \mathrm{min}$, and from 1100 to $1200{ }^{\circ} \mathrm{C}$ at $100{ }^{\circ} \mathrm{C} / \mathrm{min}$, and finally held at $1200{ }^{\circ} \mathrm{C}$ for $30 \mathrm{~min}$ under $70 \mathrm{MPa}$ pressure. Then, the SPS was turned off and samples rapidly cooled to temperatures below $400{ }^{\circ} \mathrm{C}$ under flowing argon.

Figure 13 shows the change of apparent density (determined with standard deviation less than $0.004 \times 10^{3}$ $\mathrm{kg} / \mathrm{m}^{3}$ ) and open porosity of SPS powders with increased amounts of added colloidal silica. Silverfunctionalized aerogel with the organic moiety was consolidated to density $2.63 \times 10^{3} \mathrm{~kg} / \mathrm{m}^{3}$ and contained $2.9 \%$ open porosity (SPS-1). Removal of the organic moiety before sintering (SPS-2) resulted in the increased density $2.78 \times 10^{3} \mathrm{~kg} / \mathrm{m}^{3}$ and decreased open porosity to $2.2 \%$. An addition of 5.6 (SPS3) and 10.5 (SPS-4) mass\% colloidal silica further increased density to $2.87 \times 10^{3}$ and $2.89 \times 10^{3} \mathrm{~kg} / \mathrm{m}^{3}$, and decreased open porosity to 0.5 and $0.7 \%$, respectively. Figure 14 details fused silica grains for sample SPS-3 including regions 1-4 that were used for elemental analysis. Concentrations of Ag, S, Si, and $\mathrm{O}$ in mass $\%$ for these regions are summarized in Table 4. Figure 15 shows detailed backscattered electron images of small areas of all SPS samples. Adding of colloidal silica significantly restricted accumulation of $\mathrm{Ag}$ and $\mathrm{Ag}_{2} \mathrm{~S}$ at the grain boundaries and resulted in a more uniform distribution of particles throughout the samples. Figure 16 shows pressure and ram displacement as a function of temperature. The ram displacement curves for samples SPS-1 and SPS-2 exhibited the same trend, showing a gradual increase of ram travel starting from $1060{ }^{\circ} \mathrm{C}$. However, for samples SPS-3 and SPS-4 the ram travel had already started at $880{ }^{\circ} \mathrm{C}$. This suggests that the sintering temperature of $1200{ }^{\circ} \mathrm{C}$ for $\mathrm{Ag}^{0}$-functionalized silica aerogel is too high and can be substantially reduced, i.e., for samples with or without organic moiety by more than $100{ }^{\circ} \mathrm{C}$ and for samples with added colloidal silica by more than $250{ }^{\circ} \mathrm{C}$. These results assume there is an instantaneous response between the measured temperature and the ram displacement; lower processing temperature than these may be possible.

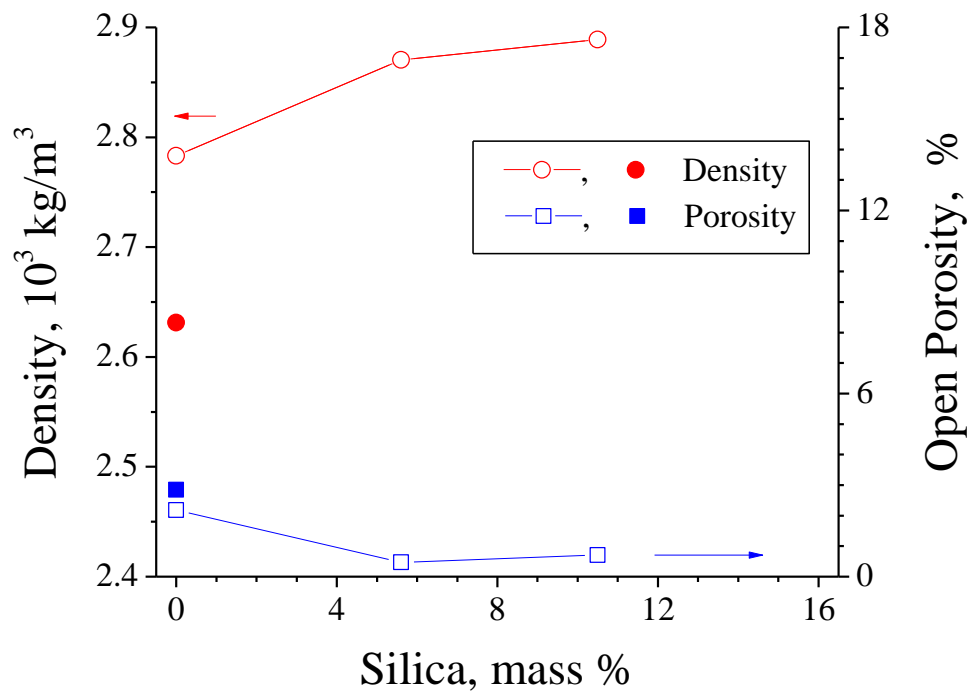


Figure 13. Apparent density and open porosity of SPS samples vs. increased concentration of added colloidal silica. Red-filled circle and blue-filled square represent sample SPS-1 ( $\mathrm{Ag}^{0}$-functionalized aerogel with organic moiety). Error bars were too small to be displayed.

A

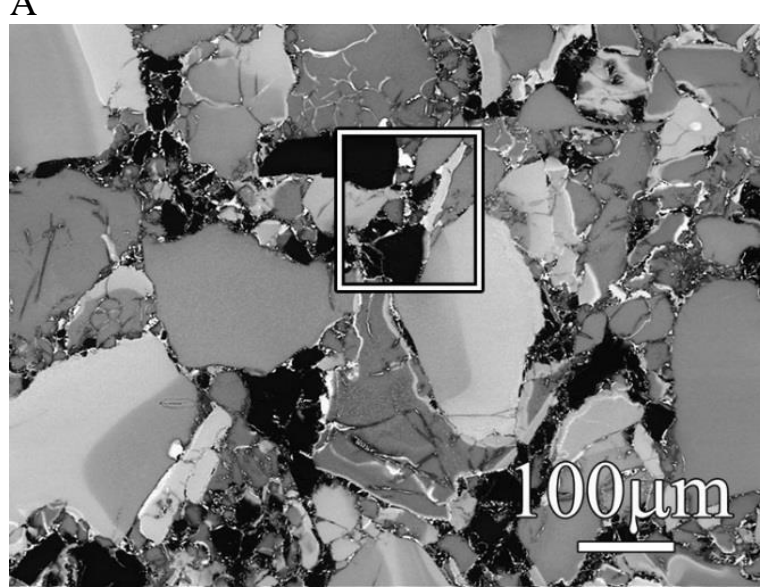

B

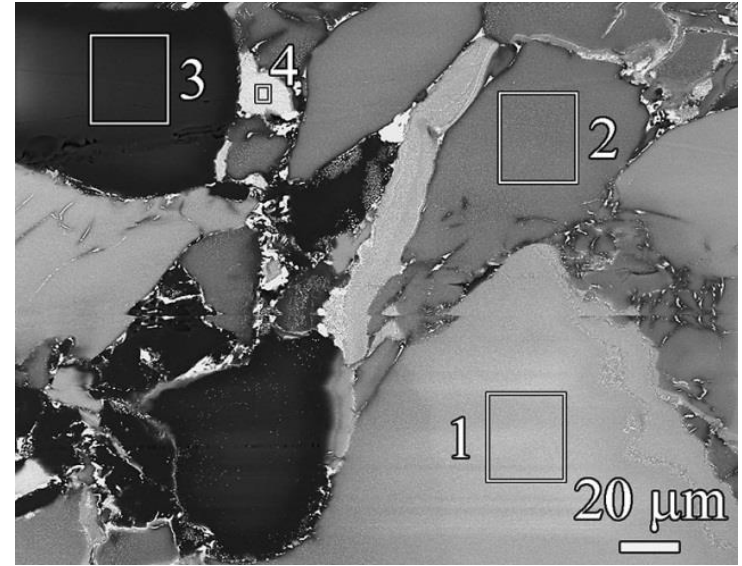

Figure 14. Backscattered electron images of fused silica grains for sample SPS-3; image B details a small square area highlighted in image A and shows regions 1-4 used for elemental analysis (summarized in Table 4).

Table 4. Concentrations of Ag, S, Si, and O in mass\% for areas from Figure 14B (SPS-3, areas 1-4).

\begin{tabular}{|c|c|c|c|c|}
\hline \multirow{2}{*}{ Area } & \multicolumn{4}{|c|}{ Concentration, mass\% } \\
\cline { 2 - 5 } & $\mathrm{Si}$ & $\mathrm{Ag}$ & $\mathrm{S}$ & $\mathrm{O}$ \\
\hline 1 & 25.97 & 27.59 & 5.43 & 41.01 \\
\hline 2 & 29.41 & 20.92 & 4.44 & 45.22 \\
\hline 3 & 34.34 & 3.35 & 1.54 & 60.78 \\
\hline 4 & 28.00 & 23.73 & 4.76 & 43.51 \\
\hline
\end{tabular}
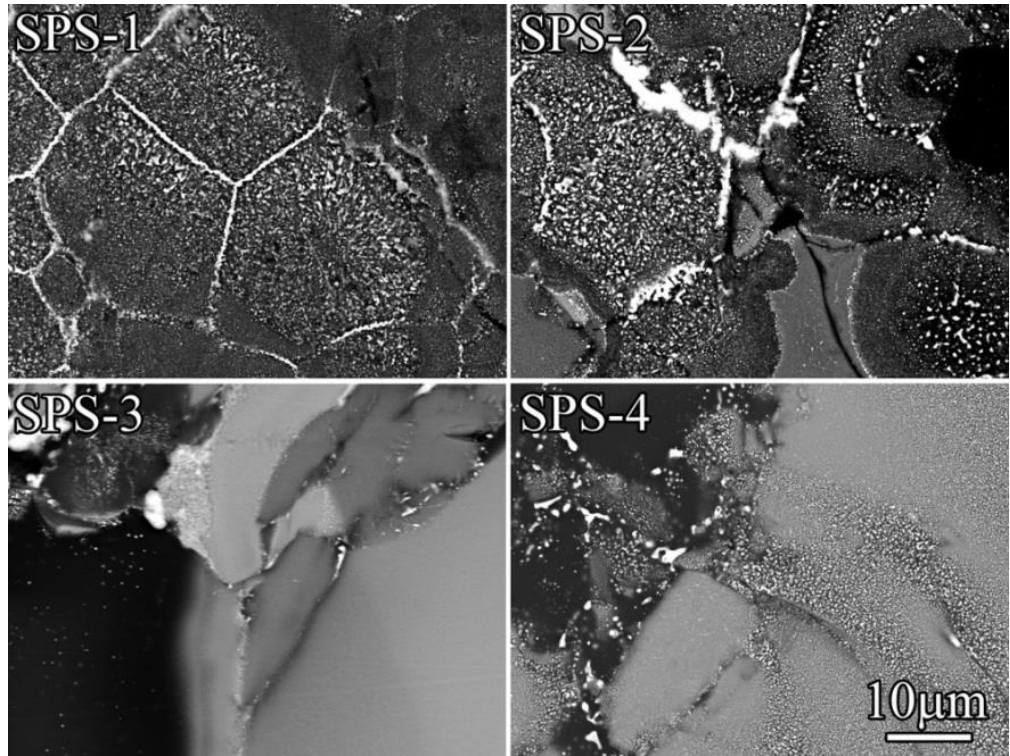

Figure 15. Backscattered electron images of fused silica grains for all SPS samples; white spots are nanoparticles and $\mu \mathrm{m}$-sized nodules of silver. 


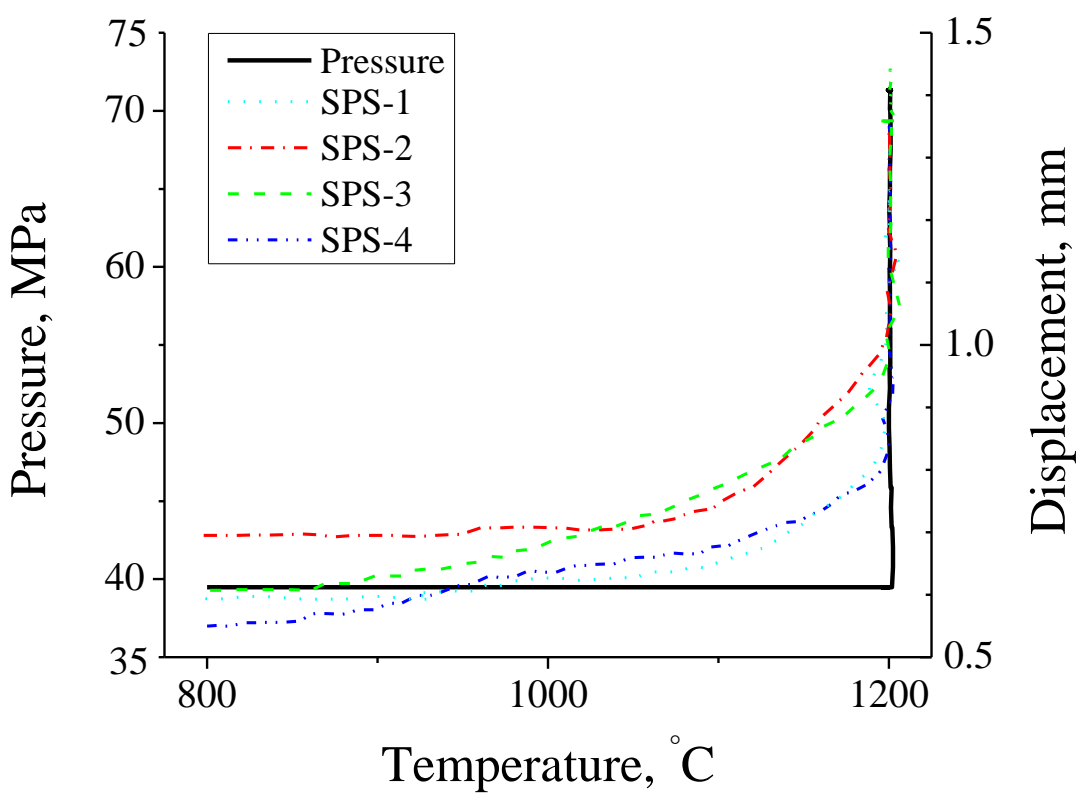

Figure 16. Pressure and ram displacement (broken lines) as functions of temperature for SPS samples.

\section{DISCUSSION}

Hot pressing, cold pressing followed by sintering in a furnace, and regular melting in a furnace are the common methods to consolidate aerogels. In contrast, there are no studies reported in the literature on the sintering of aerogels with HIP and SPS. However, the need to consolidate iodine-loaded $\mathrm{Ag}^{0}$-functionalized silica-aerogels to a viable waste form may require techniques that allow rapid consolidation at relatively low temperatures. Therefore, a preliminary investigation into the feasibility of HIP or SPS to consolidate $\mathrm{Ag}^{0}$-functionalized silica aerogel powders into a fully dense product was needed. The HUP technique was included for comparison purposes. Since these experiments were carried out at vendor facilities and in vendor equipment and the behavior of iodine during consolidation in these apparatuses was unknown, a conservative approach was taken in which no iodine was present in samples for initial testing. These preliminary experiments were designed to provide answers for the following four questions:

1. Does the organic moiety need to be removed from the pores surfaces before high-temperature densification?

2. Does additional silica promote the sintering process?

3. Do HUP, HIP, and SPS provide near-theoretically dense products at $<1200{ }^{\circ} \mathrm{C}$ ?

4. Can the sintering temperature be substantially reduced?

The results from SPS testing show marginal benefit from the removal of the organic moiety before sintering. A denser product $\left(2.78 \mathrm{vs} .2 .63 \times 10^{3} \mathrm{~kg} / \mathrm{m}^{3}\right)$ with a smaller amount of open porosity $(2.2 \mathrm{vs}$. $2.9 \%$ ) was produced from powders that did not contain organic material. Unfortunately, an attempt to HIP a sample with the same composition (no added $\mathrm{SiO}_{2}$ ) failed when the Ta envelope cracked.

However, it is a reasonable to assume that gases would have evolved from decomposition of organic moiety in the material inside the metal container and thereby prevented full densification of the powder.

Addition of small quantities of colloidal silica resulted in denser products. Adding 5.6 mass \% colloidal silica to $\mathrm{Ag}^{0}$-functionalized silica aerogel powders before processing was found to be an optimum in this initial study and provided near-theoretically dense products for HUP and SPS samples. It is assumed that this would be the case for samples from the HIP in spite of the contamination of their top surfaces with Ta. The effect of the material-container interaction was not a part of this initial study. 
The ram travel data for SPS samples clearly showed that dense pellets of $\mathrm{Ag}^{0}$-functionalized silica aerogel can be produced at temperatures well below $1200{ }^{\circ} \mathrm{C}$. For example, an addition of 5.6 mass $\%$ silica to $\mathrm{Ag}^{0}$-functionalized silica aerogel powder can decrease the sintering temperature to less than $950{ }^{\circ} \mathrm{C}$. This information is quite beneficial for our future tests with iodine-loaded aerogel, considering that lower sintering temperatures may increase the retention of iodine.

\section{CONCLUSIONS}

The preliminary investigation of HUP, HIP, and SPS clearly shows that these sintering methods can be used effectively to consolidate powders of $\mathrm{Ag}^{0}$-functionalized silica aerogel into products of neartheoretical density. The SPS technique was found to be quite effective in producing pellets with densities $>99 \%$ of theoretical. Removal of the organic moiety together with addition of small quantities of colloidal silica enhanced the sintering process and resulted in denser products. The knowledge gained from this study will be used in the next phase of the project, which focuses on identifying conditions under which the iodine-loaded aerogel is consolidated into a dense highly iodine-loaded waste form.

\section{ACKNOWLEDGEMENTS}

The authors would like to thank Jarrod Crum and Brian Riley for high-resolution SEM-EDS investigations. Robert Engler is grateful to the U.S. Department of Energy (DOE) Science Undergraduate Laboratory Internship Program for his appointment at PNNL. This work was funded by the U.S. Department of Energy's Fuel Cycle Research and Development Program. PNNL is operated for DOE by Battelle Memorial Institute under Contract DE-AC05-76RL01830.

\section{REFERENCES}

R. Jubin, N.R. Soelberg, D.M. Strachan, G. Ilas, Fuel age impacts on gaseous fission product capture during separations, FCRD-SWF-2012-000089, Oak Ridge National Laboratory, Oak Ridge, TN, 2012.

J. Matyáš, G. E. Fryxell, B. J. Busche, K. Wallace, and L. S. Fifield, Functionalized silica aerogels: Advanced materials to capture and immobilize radioactive iodine, in Ceramic Materials for Energy Applications (Eds. H. Lin, Y. Katoh, K. M. Fox, I. Belharouak, S. Widjaja, and D. Singh), John Wiley \& Sons, Inc., Hoboken, NJ, US, doi: 10.1002/9781118095386.ch3, Ceramic Engineering and Science, 32 (9), 23-33, 2011.

J. Matyáš, M. J. Robinson, and G. E. Fryxell, The effect of temperature and uniaxial pressure on the densification behavior of silica aerogel granules, in Ceramic Materials for Energy Applications II (Eds. K. Fox, Y. Katoh, H.-T. Lin, I. Belharouak, M. Halbig and S. Mathur), John Wiley \& Sons, Inc., Hoboken, NJ, USA. doi: 10.1002/9781118217535.ch9, Ceramic Engineering and Science, 33 (9), 121-133, 2012.

D. M. Strachan, J. Chun, J. Matyáš, W.C. Lepry, B.J. Riley, J. V. Ryan, and P. K. Thallapally, Summary report on the volatile radionuclide and immobilization research for FY2011 at PNNL, FCRD-SWF-2011000378, PNNL-20807, September 2011.

N. Soelberg and T. Watson, Deep bed adsorption testing using silver-functionalized aerogel, FCRDSWF-2012-000173, INL/EXT-12-26522, June 29, 2012.

S. H. Bruffey, K. K. Anderson, R. T. Jubin, and J. F. Walker Jr., Aging and iodine loading of silverfunctionalized aerogels, FCRD-SWF-2012-000256, August 31, 2012.

J. Matyáš, G. E. Fryxell, and M. J. Robinson, Characterization of dry-air aged granules of silverfunctionalized silica aerogel, FCRD-SWF-2012-000214, 2012a.

ASTM C693-93, Standard Test Method for Density of Glass by Buoyancy (2008). 
Alcoa Howmet Hot Isostatic Pressing, http://www.alcoa.com/howmet/en/info_page/desc_hip.asp

F. Zhang, M. Reich, O. Kessler, and E. Burkel, The potential of rapid cooling spark plasma sintering for metallic materials, Materials Today, Volume 16, Issue 5, May 2013, Pages 192-197, ISSN 1369-7021, http://dx.doi.org/10.1016/j.mattod.2013.05.005.

(http://www.sciencedirect.com/science/article/pii/S1369702113001521) 